\title{
Diversité des plantes mellifères de la zone soudano-guinéenne: cas de l'arrondissement de Manigri (Centre-Ouest du Bénin)
}

\author{
Hounnankpon YÉDOMONHAN ${ }^{1 *}$, Monique G. TOSSOU ${ }^{1}$, Akpovi AKOÈGNINOU ${ }^{1}$, \\ Boris B. DEMÈNOU ${ }^{1}$ et Dossahoua TRAORÉ ${ }^{2}$ \\ ${ }^{1}$ Laboratoire de Botanique et d'Ecologie végétale, Faculté des Sciences et Techniques, \\ Université d'Abomey-Calavi, 01 BP 4521 Cotonou, Bénin. \\ ${ }^{2}$ Laboratoire de Botanique, UFR Biosciences, Université de Cocody, 22 BP 582 Abidjan, Côte d'Ivoire. \\ "Auteur correspondant, E-mail : h.yedo@yahoo.fr; Tél. (00229) 95564054.
}

\section{RÉSUMÉ}

La flore mellifère environnant un rucher de l'arrondissement de Manigri a été étudiée à l'aide de relevés phénologiques et apicoles qui sont réalisés une fois par mois de janvier à décembre 2006. Ces relevés sont exécutés dans une aire d'observation de $1 \mathrm{~km}$ de rayon autour du rucher constitué de 10 ruches kenyanes. La flore mellifère est composée de 87 espèces, soit $32,7 \%$ des espèces en fleurs au nombre de 266 . Elle comporte 75 genres et 34 familles. Les familles les plus riches en espèces mellifères sont les Leguminosae (16 espèces, soit $18,4 \%$ ), les Combretaceae et les Rubiaceae (8 espèces chacune, soit 9,2\%). Les plantes mellifères sont constituées de 60 espèces d'arbres et arbustes et de 27 espèces d'herbacées. Elles renferment $31 \%$ de plantes nectarifères, $33,3 \%$ de plantes pollinifères et $35,6 \%$ de plantes productrices de nectar et de pollens.

(c) 2009 International Formulae Group. All rights reserved.

Mots clés: Diversité, flore mellifère, Manigri, zone soudano-guinéenne

\section{INTRODUCTION}

L'Afrique de l'Ouest en général, et le Bénin en particulier, ont une longue tradition apicole mais leurs flores mellifères restent encore mal connues. Il est alors rare de trouver des indications portant sur l'intérêt mellifère des différentes espèces de la flore ouest-africaine (Villières, 1987). Les plantes mellifères sont souvent identifiées à partir des analyses polliniques d'échantillons de miel (Agwu et Akambi, 1985; Lobreau-Callen et al., 1986; Millogo-Rassolodimby, 1989). Mais depuis quelques années, des travaux sur l'inventaire visuel de ces espèces sont réalisés et les plus remarquables en Afrique de l'Ouest sont ceux de: Guinko et al. (1987, 1992a, 1992b), Sawadogo (1993) et Nombré (2003), etc.

Au Bénin, depuis 2001, un programme de recherche sur les plantes mellifères et les miels a été initié au Laboratoire de Botanique et d'Ecologie Végétale de l'Université d'Abomey-Calavi. Les premiers travaux de terrain qui ont porté sur la zone sud du Bénin où règne un climat subéquatorial, ont donné lieu à deux mémoires (Fohounfo, 2002; Yédomonhan, 2004) et à une publication (Tossou et al., 2005).

Le présent travail porte sur la zone soudano-guinéenne, notamment dans l'arrondissement de Manigri. Son objectif est de déterminer la richesse en plantes mellifères de la zone en vue du développement de l'apiculture moderne.

\section{MÉTHODE D'ÉTUDE \\ Milieu d'étude}

L'arrondissement de Manigri est situé entre $8^{\circ} 35^{\prime}$ et $8^{\circ} 52^{\prime}$ de latitude Nord et $1^{\circ} 42^{\prime}$ et $2^{\circ} 15^{\prime}$ de longitude Est (figure 1 ). 
Le climat est de type soudano-guinéen (Akoègninou, 2006), caractérisé suivant les années, tantôt par quatre saisons (deux pluvieuses et deux sèches), tantôt par deux saisons (une pluvieuse et une sèche). Le total pluviométrique annuel est en moyenne de $1206 \mathrm{~mm}$. La hauteur moyenne mensuelle d'eau varie de 7,1 $\mathrm{mm}$ en janvier à $223 \mathrm{~mm}$ en août. La température moyenne mensuelle oscille entre $24^{\circ} \mathrm{C}$ en juillet et $29^{\circ} \mathrm{C}$ en mars.

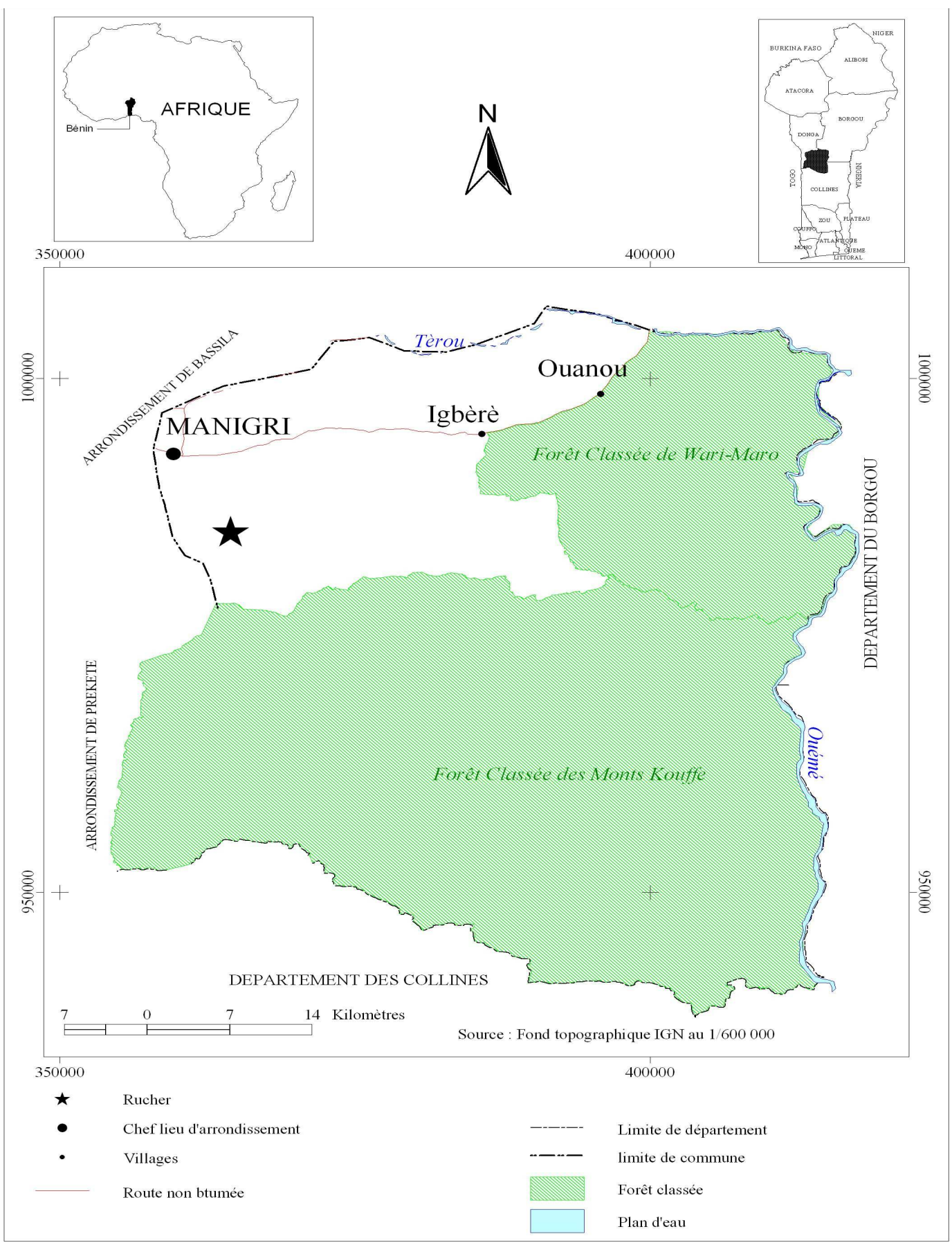

Figure 1: Localisation du rucher dans l'arrondissement de Manigri. 
Les sols sont de types ferrugineux tropicaux (Faure, 1977; Dubroeucq, 1977).

La végétation est une mosaïque de savanes, de forêts claires parsemée de galeries forestières, de champs et de plantations (Akoègninou, 2006).

La population de Manigri est estimée à 16688 habitants (Anonyme, 2003). Elle est dominée par le groupe ethnique Nago. Les activités pratiquées par cette population, par ordre d'importance, sont: l'agriculture, l'élevage de volailles et de petits ruminants, l'apiculture, le commerce, la pêche et l'exploitation forestière (Houinato, 2001).

\section{Collecte de données}

Les plantes mellifères et celles en fleurs ont été inventoriées par mois, de janvier à décembre 2006. D'après de nombreux travaux de recherche, l'aire de butinage de $A$. mellifera est fonction de la structure de la végétation et varie de 0,5 à $3 \mathrm{~km}$ de rayon (Seeley, 1985; Briane, 1991; Kepaletswe, 1997). Les travaux d'inventaire visuel des plantes mellifères réalisés dans la forêt classée de la Lama au Bénin (Yédomonhan, 2004) et d'analyse pollinique des miels récoltés dans ce même cadre (Tossou et al., 2005) ont montré que seulement $1,4 \%$ de la flore mellifère est situé en dehors du rayon de $1 \mathrm{~km}$ autour du rucher. Par conséquent, le périmètre d'inventaires floristiques adopté est de $1 \mathrm{~km}$ autour du rucher expérimental, constitué de 10 ruches kenyanes.

Les inventaires ont été exécutés dans des placeaux de $500 \mathrm{~m}^{2}(20 \mathrm{~m} \times 25 \mathrm{~m})$, installés à tous les $200 \mathrm{~m}$ dans l'aire de butinage de Apis mellifera adansonii Latr. Les espèces en fleurs et les espèces butinées par les abeilles sont enregistrées. Le nutriment recherché par l'insecte est identifié à l'œil nu ou à l'aide d'une paire de jumelles au besoin, sur la base du comportement d'affouragement de l'abeille butineuse. Lorsqu'il s'agit d'une plante seulement pollinifère, l'abeille s'arrête uniquement au niveau des anthères et confectionne des pelotes de pollens bien visibles par leur couleur, logées dans les corbeilles des pattes postérieures. Mais, quand il est question de plante nectarifère, l'insecte enfonce sa trompe dans la fleur pour y sucer le nectar. Quant aux plantes à la fois pollinifères et nectarifères, on observe les deux comportements chez une même butineuse; ou bien elle prélève seulement le pollen ou le nectar.

Le taux de butinage est estimé en pourcentage de fleurs visitées par les abeilles par rapport à l'ensemble des fleurs de chaque plante.

\section{Analyse et traitement des données}

La richesse spécifique est établie pour les plantes mellifères et aussi pour les plantes en fleurs. La nomenclature botanique utilisée est celle de Akoègninou et al. (2006).

Les types biologiques considérés sont ceux de Ramirez (2002) à savoir: arbres, arbustes et herbacées.

Suivant la durée de floraison, 3 classes de floraison sont définies : Classe I : un mois de floraison ; Classe II : un mois à deux mois continus de floraison; Classe III : plus de deux mois de floraison (Guinko et al., 1992a).

Le taux de butinage (t) maximal étant estimé à $30 \%$, les 3 différentes classes d'espèces mellifères, en tenant compte de l'intensité de butinage, sont: Classe A : espèces faiblement butinées $(0<\mathrm{t}<5 \%)$; Classe $\mathrm{B}$ : espèces moyennement butinées $(5 \leq \mathrm{t}<10 \%)$; Classe $\mathrm{C}$ : espèces intensément butinées $(10 \leq \mathrm{t} \leq 30 \%)$.

La nature et le degré de corrélation entre la richesse spécifique mensuelle des plantes en fleurs et celles de plantes mellifères sont évalués avec le coefficient de corrélation de Pearson (r) grâce au logiciel MINITAB 13.20. Le test de signification de $r$ est fait à base du tableau des valeurs critiques. Les valeurs critiques dans ce cas $(n=12$ relevés mensuels ou degré de liberté $n-2=10)$ sont de 0,576 au seuil de probabilité $\mathrm{p}<0,05$ et de 0,708 à $\mathrm{p}<0,01$.

\section{RÉSULTATS}

\section{Diversité floristique des plantes mellifères}

Les observations de butinage d'Apis mellifera adansonii Lat. autour du rucher ont permis de recenser 87 espèces mellifères sur 266 espèces en fleurs, soit un taux de sélection de $32,7 \%$.

Les espèces mellifères se composent de 28 arbres (soit 32,2\%), 32 arbustes (soit $36,8 \%$ ) et 27 espèces herbacées (soit $31 \%$ ). Elles se regroupent en 34 familles et 75 genres (tableau 1). Les Leguminosae sont les plus visitées avec 16 espèces, soit 18,4\% dont $10,3 \%$ pour les Caesalpinioideae, $4,6 \%$ pour 
les Mimosoideae et $3,5 \%$ pour les Papilionoideae. Elles sont suivies des Combretaceae et des Rubiaceae, avec chacune 8 espèces (9,2\%); viennent ensuite les Euphorbiaceae, avec 6 espèces, soit $6,9 \%$ de la flore mellifère.

Parmi les plantes mellifères recensées, 27 sont nectarifères (soit 31\%), 29 sont pollinifères (soit $33,3 \%$ ) et 31 sont à la fois nectarifères et pollinifères (soit 35,6\%).

En tenant compte de la durée de floraison, trois classes d'espèces mellifères sont obtenues. La classe I regroupant les espèces dont la durée de floraison est d'un mois et la classe III réunissant les taxons dont la durée de floraison est supérieure à deux mois, viennent en tête avec 30 espèces chacune, soit $34,5 \%$ de la flore ligneuse mellifère. Enfin, suit la classe II qui comporte 27 espèces, soit $31 \%$ et dont la durée de floraison des espèces s'étale sur deux mois successifs.

Suivant le taux de butinage, trois classes d'espèces sont identifiées. La classe A des espèces faiblement butinées est la plus fournie en taxons (39 espèces) dont 18,4\% d'espèces pollinifères, $14,9 \%$ d'espèces nectarifères et $11,5 \%$ d'espèces productrices de nectar et de pollens. La classe B des espèces moyennement butinées est moins diversifiée ; elle compte 23 taxons, soit $26,4 \%$ de la flore mellifère dont 12 espèces (soit $13,8 \%$ ) à la fois nectarifères et pollinifères, 6 espèces pollinifères (soit $6,9 \%$ ) et 5 espèces nectarifères (soit 5,7\%). La classe C des espèces intensément butinées comprend 25 taxons, soit $28,7 \%$ de la flore mellifère dont 9 espèces à la fois nectarifères et pollinifères (soit 10,3\%), 9 espèces nectarifères (soit $10,3 \%$ ) et 7 espèces pollinifères (soit $8,1 \%$ ).

En combinant la durée de floraison et le taux de butinage, 16 espèces sont intensément butinées par les abeilles et disponibles pour elles pendant au moins deux mois de suite. Parmi elles, 7 soit $8,1 \%$ sont nectarifères ; il s'agit de Burkea africana, Cyphostemma adenocaule, Daniellia oliveri, Lonchocarpus sericeus, Parinari curatellifolia, Tectona grandis, Vernonia colorata), 8 autres (soit $9,2 \%$ ) sont nectarifères et pollinifères (Cissus populnea, Combretum collinum, Combretum zenkeri, Grewia mollis, Hymenocardia acida, Pteleopsis suberosa, Syzygium guineense,
Tamarindus indica) et 1 , soit $1,1 \%$, est pollinifère (Lannea barteri).

\section{Relation entre la richesse spécifique florale et la richesse spécifique mellifère}

La figure 2 montre la variation mensuelle du nombre d'espèces en fleurs et du nombre d'espèces mellifères au cours de la période d'observation.

La richesse spécifique des plantes en fleurs varie de 18 espèces en février à 76 espèces en septembre. Elle est caractérisée par une abondante floraison des arbres et arbustes entre janvier (16 espèces) et mai (24 espèces) et des herbacées entre juin (56 espèces) et décembre (68 espèces), avec le pic en septembre (71 espèces) (tableau 2). La richesse spécifique mensuelle est positivement corrélée $(\mathrm{r}=0,958 ; \mathrm{p}=0,000)$ à celle des herbacées et négativement corrélée à celles des arbres et arbustes $(r=-0,624 ; p=0,030)$. L'évolution du nombre mensuel d'espèces ligneuses (arbres et arbustes) et de celui d'herbacées met en évidence une corrélation linéaire négative et hautement significative $(\mathrm{r}=-0,821 ; \mathrm{p}=0,001)$. La richesse spécifique des plantes herbacées est donc inversement proportionnelle à celle des plantes ligneuses en fleurs.

S'agissant des plantes mellifères, le nombre d'espèces est compris entre 4 en décembre et 35 en mai. L'évolution de la richesse spécifique au cours des mois présente deux pics dont le premier en mars avec 28 espèces et le second en mai avec 35 espèces (tableau II). Les arbres et arbustes prédominent entre janvier (13 espèces) et mai (23 espèces), avec le maximum en mars (24 espèces). Par contre, la richesse spécifique en herbacées mellifères est plus importante entre juin (12 espèces) et octobre (11 espèces), avec le pic en septembre (13 espèces). Le nombre total mensuel d'espèces mellifères est positivement corrélé $(\mathrm{r}=0,841 ; \mathrm{p}=0,001)$ à la ri-chesse spécifique des arbres et arbustes. Il n'est pas, par contre, corrélé à la richesse spécifique mensuelle des herbacées $(r=0,240$; $\mathrm{p}=0,453)$. La corrélation linéaire entre le nombre mensuel d'espèces d'arbres et d'arbustes et celui des herbacées est négative mais aussi non significative $(r=-0,323 ; p=0,305)$.

L'analyse de régression linéaire simple montre que la richesse spécifique mensuelle des plantes mellifères ne présente pas une 
Tableau 1: Liste des plantes mellifères et leur période de floraison.

\begin{tabular}{|c|c|c|c|c|c|c|c|c|c|c|c|c|c|c|c|}
\hline Espèces & FB & Nut & TB & $\mathbf{J}$ & $\mathbf{F}$ & Ms & Av & Mi & Jn & $\mathbf{J l}$ & Ao & S O & $\mathbf{N} \mathbf{D}$ & CD & CI \\
\hline \multicolumn{16}{|l|}{ Anacardiaceae } \\
\hline Anacardium occidentale L. & A & $\mathrm{N}$ & 5 & $*$ & $*$ & $*$ & & & & & & & & III & $\mathrm{B}$ \\
\hline Lannea acida A.Rich. s.l. & A & $\mathrm{NP}$ & 5 & $*$ & $*$ & & & & & & & & & II & $\mathrm{B}$ \\
\hline $\begin{array}{l}\text { Lannea barteri (Oliv.) } \\
\text { Engl. } ₫\end{array}$ & a & $\mathrm{P}$ & 20 & $*$ & $*$ & & & & & & & & & II & $\mathrm{C}$ \\
\hline \multicolumn{16}{|l|}{ Annonaceae } \\
\hline Annona senegalensis Pers. & $\mathrm{a}$ & NP & 2 & & & $*$ & $*$ & $*$ & & & & & & III & A \\
\hline \multicolumn{16}{|l|}{ Araliaceae } \\
\hline $\begin{array}{l}\text { Cussonia arborea } \\
\text { Hochst. ex A.Rich. }\end{array}$ & A & $\mathrm{NP}$ & 2 & & & & & $*$ & $*$ & & & & & II & A \\
\hline \multicolumn{16}{|l|}{ Arecaceae } \\
\hline Borassus aethiopum Mart. & A & $\mathrm{P}$ & 25 & & & & $*$ & & & & & & & I & $\mathrm{C}$ \\
\hline Elaeis guineensis Jacq. & A & $\mathrm{P}$ & 10 & & & & & & & & & $*$ & & I & $\mathrm{C}$ \\
\hline \multicolumn{16}{|l|}{ Asclepiadaceae } \\
\hline $\begin{array}{l}\text { Pachycarpus lineolatus } \\
\text { (Decne.) Bullock }\end{array}$ & $\mathrm{H}$ & $\mathrm{NP}$ & 1 & & & & & & & $*$ & & & & I & A \\
\hline \multicolumn{16}{|l|}{ Asteraceae } \\
\hline Bidens bipinnata $\mathrm{L}$. & $\mathrm{H}$ & $\mathrm{P}$ & 1 & & & & & & & & & $* *$ & & II & A \\
\hline Bidens pilosa $\mathrm{L}$. & $\mathrm{H}$ & $\mathrm{P}$ & 1 & & & & & & & & & $* *$ & $*$ & III & A \\
\hline Tridax procumbens $\mathrm{L}$. & $\mathrm{H}$ & $\mathrm{P}$ & 1 & & & & & & & & & $*$ & & I & A \\
\hline $\begin{array}{l}\text { Vernonia colorata } \\
\text { (Willd.) Drake } a\end{array}$ & $\mathrm{a}$ & $\mathrm{N}$ & 10 & $*$ & $*$ & & & & & & & & & II & $\mathrm{C}$ \\
\hline \multicolumn{16}{|l|}{ Balanophoraceae } \\
\hline Thonningia sanguinea $\mathrm{Vahl}$ & $\mathrm{H}$ & $\mathrm{P}$ & 5 & & & & & & & $*$ & $*$ & & & II & $\mathrm{B}$ \\
\hline \multicolumn{16}{|l|}{ Bignoniaceae } \\
\hline $\begin{array}{l}\text { Stereosperpum } \\
\text { kunthianum Cham. }\end{array}$ & A & $\mathrm{N}$ & 2 & $*$ & & & & & & & & & & I & A \\
\hline \multicolumn{16}{|l|}{ Celtidaceae } \\
\hline $\begin{array}{l}\text { Trema orientalis }(\mathrm{L} .) \\
\text { Blume }\end{array}$ & $\mathrm{a}$ & $\mathrm{N}$ & 5 & & & & & $*$ & & & & & & I & B \\
\hline \multicolumn{16}{|l|}{ Chrysobalanaceae } \\
\hline $\begin{array}{l}\text { Maranthes polyandra } \\
\text { (Benth.) Prance }\end{array}$ & $\mathrm{a}$ & $\mathrm{P}$ & 5 & & & & & * & & $*$ & & & & II & $\mathrm{B}$ \\
\hline $\begin{array}{l}\text { Parinari curatellifolia } \\
\text { Planch. ex Benth. } \times\end{array}$ & $\mathrm{a}$ & $\mathrm{N}$ & 15 & $*$ & $*$ & $*$ & & & & & & & & III & $\mathrm{C}$ \\
\hline \multicolumn{16}{|l|}{ Clusiaceae } \\
\hline $\begin{array}{l}\text { Psorospermum } \\
\text { febrifugum Spach }\end{array}$ & $\mathrm{a}$ & $\mathrm{N}$ & 2 & & & & $*$ & $*$ & & & & & & II & A \\
\hline \multicolumn{16}{|l|}{ Combretaceae } \\
\hline $\begin{array}{l}\text { Anogeissus leiocarpa (DC.) } \\
\text { Guill. \& Perr. }\end{array}$ & A & $\mathrm{N}$ & 10 & & & & & $*$ & & & & & & I & $\mathrm{C}$ \\
\hline $\begin{array}{l}\text { Combretum adenogonium } \\
\text { Steud. ex A.Rich. }\end{array}$ & a & $\mathrm{NP}$ & 2 & $*$ & $*$ & $*$ & & & & & & & & III & A \\
\hline $\begin{array}{l}\text { Combretum collinum } \\
\text { Fresen. } ם\end{array}$ & $\mathrm{a}$ & $\mathrm{NP}$ & 15 & $*$ & $*$ & $*$ & $*$ & & & & & & & III & $\mathrm{C}$ \\
\hline
\end{tabular}




\begin{tabular}{|c|c|c|c|c|c|c|c|c|c|c|c|c|c|c|c|c|}
\hline $\begin{array}{l}\text { Combretum racemosum } \\
\text { P. Beauv. }\end{array}$ & $\mathrm{a}$ & NP & 5 & $*$ & & & & & & & & & & & I & B \\
\hline $\begin{array}{l}\text { Combretum zenkeri } \\
\text { Engl. \& Diels } \propto\end{array}$ & $\mathrm{a}$ & NP & 10 & & & & & & & & & & $*$ & & II & $\mathrm{C}$ \\
\hline $\begin{array}{l}\text { Pteleopsis suberosa } \\
\text { Engl. \& Diels }\end{array}$ & A & NP & 10 & $*$ & $*$ & & & & & & & & & $* *$ & III & $\mathrm{C}$ \\
\hline $\begin{array}{l}\text { Terminalia avicennoides } \\
\text { Guill. \& Perr. }\end{array}$ & $\mathrm{a}$ & NP & 10 & & & $*$ & $*$ & $*$ & & & & & & & III & $\mathrm{B}$ \\
\hline $\begin{array}{l}\text { Terminalia glaucescens } \\
\text { Planch. ex Benth. }\end{array}$ & a & NP & 5 & & & & $*$ & & & & & & & & I & $\mathrm{C}$ \\
\hline \multicolumn{17}{|l|}{ Commelinaceae } \\
\hline Commelina erecta $\mathrm{L}$. & $\mathrm{H}$ & $\mathrm{P}$ & 1 & & & $*$ & & $*$ & $*$ & $*$ & $*$ & $*$ & $*$ & $* *$ & III & A \\
\hline Cyanotis lanata Benth. & $\mathrm{H}$ & $\mathrm{P}$ & 5 & & & & & & & & & $*$ & $*$ & & II & $\mathrm{B}$ \\
\hline \multicolumn{17}{|l|}{ Connaraceae } \\
\hline $\begin{array}{l}\text { Rourea coccinea (Thonn.ex } \\
\text { Schumach.) Benth. }\end{array}$ & $\mathrm{H}$ & $\mathrm{N}$ & 5 & & & $*$ & & $*$ & & $*$ & $*$ & $*$ & $*$ & & III & $\mathrm{B}$ \\
\hline \multicolumn{17}{|l|}{ Cyperaceae } \\
\hline $\begin{array}{l}\text { Bulbostylis pilosa (Willd.) } \\
\text { Cherm. }\end{array}$ & $\mathrm{H}$ & $\mathrm{P}$ & 2 & & & & & $*$ & $*$ & $*$ & $*$ & $*$ & $*$ & & III & A \\
\hline \multicolumn{17}{|l|}{ Dipterocarpaceae } \\
\hline Monotes kerstingii Gilg & A & $\mathrm{P}$ & 2 & & & $*$ & & & & & & & & & I & A \\
\hline \multicolumn{17}{|l|}{ Euphorbiaceae } \\
\hline $\begin{array}{l}\text { Antidesma venosum } \\
\text { E. Mey. ex Tul. }\end{array}$ & a & $\mathrm{N}$ & 7 & & & & & $*$ & & & & & & & I & $\mathrm{B}$ \\
\hline Bridelia ferruginea Benth. & $\mathrm{a}$ & $\mathrm{N}$ & 2 & & & & $*$ & $*$ & $*$ & & & & & & III & A \\
\hline $\begin{array}{l}\text { Flueggea virosa (Roxb. } \\
\text { ex Willd.) Voigt }\end{array}$ & $\mathrm{H}$ & $\begin{array}{l}\mathrm{N} \\
\mathrm{P}\end{array}$ & 5 & & & & & $*$ & $*$ & $*$ & $*$ & & & & III & B \\
\hline Hymenocardia acida Tul. ฉ & $\mathrm{a}$ & $\begin{array}{l}\mathrm{N} \\
\mathrm{P}\end{array}$ & 10 & & & $*$ & $*$ & $*$ & & & & & & & III & $\mathrm{C}$ \\
\hline $\begin{array}{l}\text { Margaritaria discoidea } \\
\text { (Baill.) Webster }\end{array}$ & A & $\mathrm{P}$ & 10 & & & & $*$ & & & & & & & & I & $\mathrm{C}$ \\
\hline Phyllanthus reticulatus Poir. & $\mathrm{a}$ & $\mathrm{P}$ & 1 & & & & & $*$ & $*$ & & & & & & II & A \\
\hline \multicolumn{17}{|l|}{ Flacourtiaceae } \\
\hline $\begin{array}{l}\text { Flacourtia indica } \\
\text { (Burm.f.) Merr. }\end{array}$ & a & $\begin{array}{l}\mathrm{N} \\
\mathrm{P}\end{array}$ & 5 & & & $*$ & & & & & & $*$ & & & II & B \\
\hline \multicolumn{17}{|l|}{$\begin{array}{l}\text { Leguminosae- } \\
\text { Caesalpinioideae }\end{array}$} \\
\hline $\begin{array}{l}\text { Berlinia grandiflora (Vahl) } \\
\text { Hutch. \& Dalziel }\end{array}$ & A & $\mathrm{P}$ & 3 & & & $*$ & $*$ & & & & & & & & II & A \\
\hline Burkea africana Hook. x & $\mathrm{a}$ & $\mathrm{N}$ & 10 & & & $*$ & $*$ & $*$ & & & & & & & III & $\mathrm{C}$ \\
\hline Cassia sieberiana DC. & $\mathrm{a}$ & $\mathrm{P}$ & 10 & & & $*$ & & & & & & & & & I & $\mathrm{C}$ \\
\hline $\begin{array}{l}\text { Chamaecrista } \\
\text { mimosoides (L.) Greene }\end{array}$ & $\mathrm{H}$ & $\begin{array}{l}\mathrm{N} \\
\mathrm{P}\end{array}$ & 3 & & & & & & & & & $*$ & $*$ & & II & A \\
\hline $\begin{array}{l}\text { Daniellia oliveri (Rolfe) } \\
\text { Hutch. \& Dalziel } \propto\end{array}$ & A & $\mathrm{N}$ & 20 & $*$ & $*$ & & & & & & & & & & II & $\mathrm{C}$ \\
\hline $\begin{array}{l}\text { Detarium microcarpum } \\
\text { Guill. \& Perr. }\end{array}$ & $\mathrm{a}$ & $\begin{array}{l}\mathrm{N} \\
\mathrm{P}\end{array}$ & 5 & & & & & $*$ & & & & & & & I & B \\
\hline $\begin{array}{l}\text { Erythrophleum suaveolens } \\
\text { (Guill. \& Perr.) Br. }\end{array}$ & A & $\mathrm{P}$ & 30 & & $*$ & $*$ & & & & & & & & & I & $\mathrm{C}$ \\
\hline $\begin{array}{l}\text { Isoberlinia doka } \\
\text { Craib \& Stapf }\end{array}$ & A & $\mathrm{N}$ & 4 & & $*$ & & & & & & & & & & I & A \\
\hline Tamarindus indica L. ø & A & $\begin{array}{l}\mathrm{N} \\
\mathrm{P}\end{array}$ & 10 & & & & $*$ & $*$ & & & & & & & II & $\mathrm{C}$ \\
\hline
\end{tabular}




\begin{tabular}{|c|c|c|c|c|c|c|c|c|c|c|c|c|c|c|c|}
\hline \multicolumn{16}{|l|}{$\begin{array}{l}\text { Leguminosae- } \\
\text { Mimosoideae }\end{array}$} \\
\hline Acacia polyacantha Willd. & $\mathrm{A}$ & $\mathrm{P}$ & 4 & & & & $*$ & $*$ & & & & & & II & $\mathrm{A}$ \\
\hline Acacia sieberiana DC. & A & $\mathrm{P}$ & 10 & & & & $*$ & & & & & & & I & $\mathrm{C}$ \\
\hline $\begin{array}{l}\text { Dichrostachys cinerea (L.) } \\
\text { Wight \& Arn. }\end{array}$ & $\mathrm{a}$ & $\mathrm{P}$ & 5 & & & & & $*$ & & & & & & I & $\mathrm{B}$ \\
\hline $\begin{array}{l}\text { Entada africana } \\
\text { Guill. \& Perr. }\end{array}$ & A & $\begin{array}{l}\mathrm{N} \\
\mathrm{P}\end{array}$ & 5 & & & & $*$ & & & & & & & I & $\mathrm{B}$ \\
\hline \multicolumn{16}{|l|}{$\begin{array}{l}\text { Leguminosae- } \\
\text { Papilionoideae }\end{array}$} \\
\hline $\begin{array}{l}\text { Aganope stuhlmannii } \\
\text { (Taub.) Adema }\end{array}$ & A & $\mathrm{N}$ & 2 & & $*$ & $*$ & & & & & & & & II & A \\
\hline $\begin{array}{l}\text { Lonchocarpus sericeus } \\
\text { (Poir.) Kunth } \ltimes\end{array}$ & A & $\mathrm{N}$ & 10 & & & & & & $*$ & $*$ & $*$ & & & III & $\mathrm{C}$ \\
\hline $\begin{array}{l}\text { Pericopsis laxiflora } \\
\text { (Benth. ex Baker) Meeu. }\end{array}$ & $\mathrm{a}$ & $\mathrm{N}$ & 4 & & & & $*$ & $*$ & $*$ & & & & & III & A \\
\hline \multicolumn{16}{|l|}{ Loganiaceae } \\
\hline Strychnos innocua Delile & $\mathrm{a}$ & $\mathrm{N}$ & 4 & & & $*$ & & $*$ & & & & $*$ & & III & $\mathrm{A}$ \\
\hline Strychnos spinosa Lam. & $\mathrm{a}$ & $\mathrm{N}$ & 3 & & & $*$ & & & & & & & & I & A \\
\hline \multicolumn{16}{|l|}{ Loranthaceae } \\
\hline $\begin{array}{l}\text { Agelanthus dodoneifolius } \\
\text { (DC.) Polh \& W. }\end{array}$ & $\mathrm{H}$ & $\mathrm{P}$ & 1 & $*$ & $*$ & & & & & & & & & II & A \\
\hline \multicolumn{16}{|l|}{ Malvaceae } \\
\hline Sida acuta Burm.f. ssp. acuta & $\mathrm{H}$ & $\mathrm{NP}$ & 5 & & & & & & $*$ & $*$ & $*$ & $*$ & $*$ & III & $\mathrm{B}$ \\
\hline Sida garckeana Pol. & $\mathrm{H}$ & NP & 1 & & & & & & & $*$ & $*$ & $*$ & $*$ & III & $\mathrm{A}$ \\
\hline \multicolumn{16}{|l|}{ Meliaceae } \\
\hline $\begin{array}{l}\text { Pseudocedrela kotschyi } \\
\text { (Schweinf.) Harms }\end{array}$ & A & $\mathrm{N}$ & 2 & & & $*$ & & & & & & & & $\mathrm{I}$ & A \\
\hline Trichilia emetica Vahl & $\mathrm{a}$ & NP & 5 & & & $*$ & $*$ & $*$ & & & & & & III & $\mathrm{B}$ \\
\hline \multicolumn{16}{|l|}{ Myrtaceae } \\
\hline $\begin{array}{l}\text { Syzygium guineense } \\
\text { (Willd.) DC. ฉ }\end{array}$ & A & NP & 10 & $*$ & $*$ & & & & & & & & & II & $\mathrm{C}$ \\
\hline \multicolumn{16}{|l|}{ Orchidaceae } \\
\hline Eulophia guineensis Lindl. & $\mathrm{H}$ & $\mathrm{P}$ & 1 & & & $*$ & & & & & & & & $\mathrm{I}$ & A \\
\hline \multicolumn{16}{|l|}{ Poaceae } \\
\hline $\begin{array}{l}\text { Andropogon tectorum } \\
\text { Schumach. \& Thonn. }\end{array}$ & $\mathrm{H}$ & $\mathrm{P}$ & 1 & $*$ & & & & & & & & & $* *$ & III & A \\
\hline $\begin{array}{l}\text { Brachiaria deflexa } \\
\text { (Schumach.) Robyns }\end{array}$ & $\mathrm{H}$ & $\mathrm{P}$ & 2 & & & & & $*$ & $*$ & & & & & II & A \\
\hline $\begin{array}{l}\text { Brachiaria jubata } \\
\text { (Fig. \& De Not.) Stapf. }\end{array}$ & $\mathrm{H}$ & $\mathrm{P}$ & 4 & & & & & & $*$ & & & & & $\mathrm{I}$ & A \\
\hline $\begin{array}{l}\text { Hyparrhenia smithiana } \\
\text { (Hook.f.) Stapf }\end{array}$ & $\mathrm{H}$ & $\mathrm{P}$ & 1 & & & & & & & & & & $*$ & I & A \\
\hline $\begin{array}{l}\text { Sporobolus pyramidalis } \\
\text { P. Beauv. }\end{array}$ & $\mathrm{H}$ & $\mathrm{P}$ & 5 & & & & & $*$ & $*$ & & & & & II & $\mathrm{B}$ \\
\hline \multicolumn{16}{|l|}{ Polygalaceae } \\
\hline $\begin{array}{l}\text { Securidaca longepedunculata } \\
\text { Fresen. }\end{array}$ & $\mathrm{a}$ & $\mathrm{N}$ & 2 & & & & & $*$ & & & & & & I & A \\
\hline \multicolumn{16}{|l|}{ Rubiaceae } \\
\hline $\begin{array}{l}\text { Crossopteryx febrifuga } \\
\text { (G. Don) Benth. }\end{array}$ & A & $\mathrm{NP}$ & 2 & & & & & $*$ & $*$ & & & & & II & A \\
\hline Keetia venosa (Oliv.) Bridson & $\mathrm{a}$ & $\mathrm{P}$ & 1 & & & & & & & $*$ & $*$ & & & II & A \\
\hline $\begin{array}{l}\text { Pavetta corymbosa } \\
\text { (DC.) F.N. Williams }\end{array}$ & $\mathrm{a}$ & $\mathrm{N}$ & 5 & & & $*$ & & $*$ & & & & & & $\mathrm{I}$ & $\mathrm{B}$ \\
\hline Pavetta crassipes K. Schum. & $\mathrm{H}$ & $\mathrm{N}$ & 3 & & & & & $*$ & & & & & & I & A \\
\hline
\end{tabular}




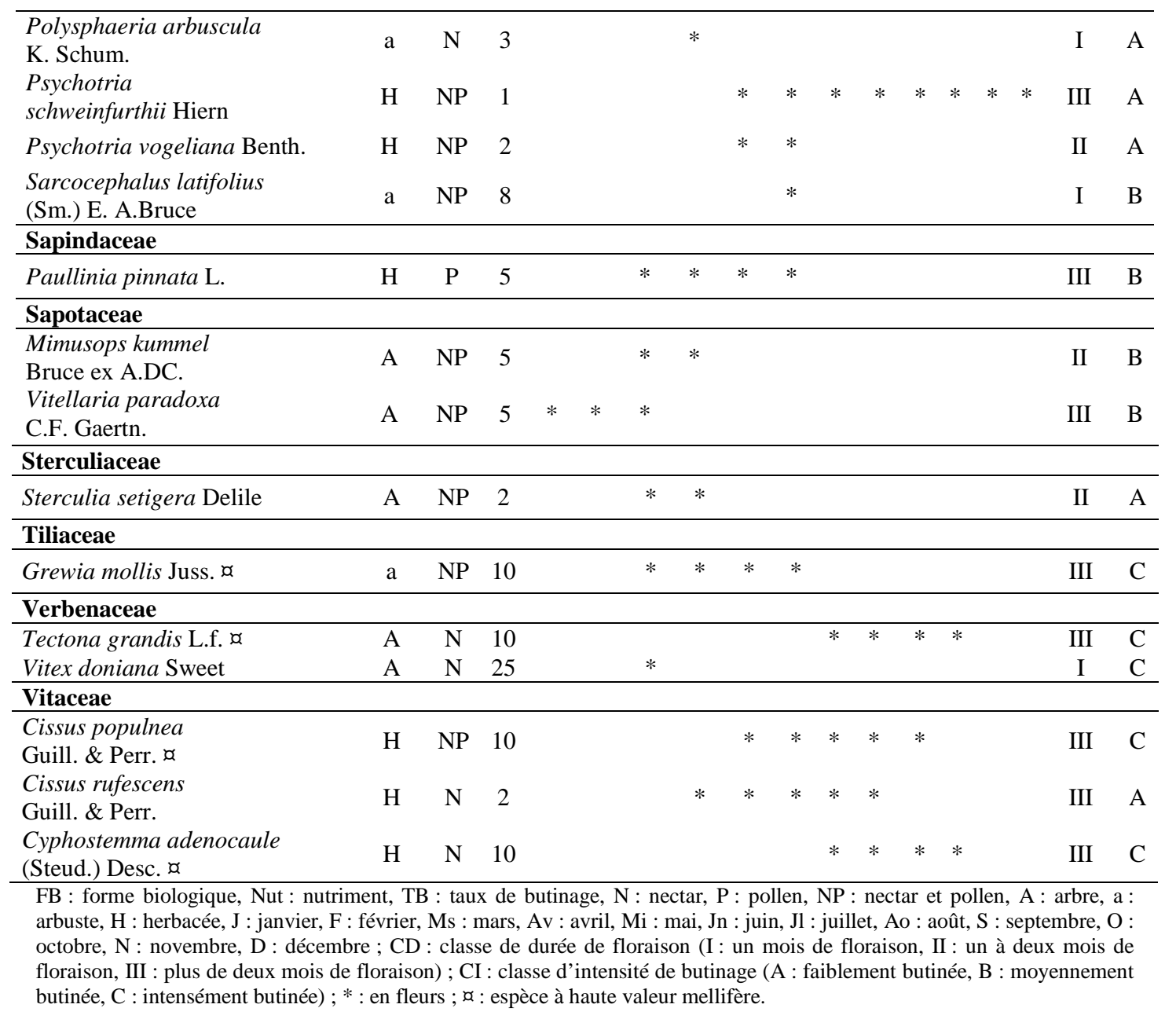

Tableau 2: Richesse spécifique mensuelle des plantes mellifères et en fleurs.

\begin{tabular}{|c|c|c|c|c|c|c|}
\hline \multirow{2}{*}{ Mois } & \multicolumn{3}{|c|}{ Espèces en fleurs } & \multicolumn{3}{|c|}{ Espèces mellifères } \\
\hline & Global & Arbres et arbustes & Herbacées & Global & Arbres et arbustes & Herbacées \\
\hline Janvier & 34 & 16 & 18 & 15 & 13 & 2 \\
\hline Février & 18 & 17 & 1 & 14 & 13 & 1 \\
\hline Mars & 47 & 27 & 20 & 28 & 24 & 4 \\
\hline Avril & 30 & 22 & 8 & 23 & 21 & 2 \\
\hline Mai & 57 & 24 & 33 & 35 & 23 & 12 \\
\hline Juin & 66 & 10 & 56 & 20 & 8 & 12 \\
\hline Juillet & 51 & 6 & 45 & 16 & 4 & 12 \\
\hline Août & 48 & 5 & 43 & 14 & 2 & 12 \\
\hline Septembre & 76 & 5 & 71 & 17 & 4 & 13 \\
\hline Octobre & 70 & 2 & 68 & 14 & 3 & 11 \\
\hline Novembre & 71 & 2 & 69 & 6 & 1 & 5 \\
\hline Décembre & 70 & 2 & 68 & 4 & 1 & 3 \\
\hline Total & 266 & 70 & 196 & 87 & 60 & 27 \\
\hline
\end{tabular}




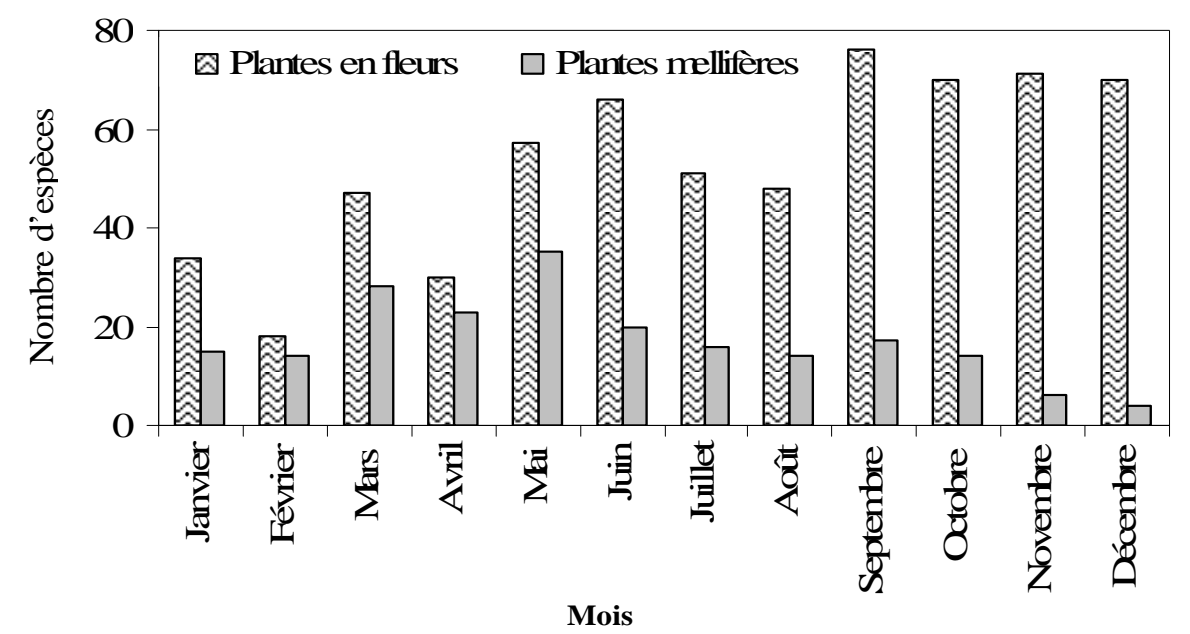

Figure 2: Evolution du nombre mensuel d'espèces en fleurs et de plantes mellifères.

corrélation significative avec la richesse spécifique mensuelle des plantes en fleurs $(\mathrm{r}=$ $-0,230 ; p=0,406)$. Seuls $5,28 \%$ de la variation du nombre mensuel d'espèces de plantes mellifères s'expliquent par la variation du nombre mensuel d'espèces de plantes en fleurs. Dans le détail, en considérant les arbres et arbustes, la richesse spécifique en plantes mellifères est fortement et positivement corrélée avec celle des plantes en fleurs $(r=$ $0,982$ et $p=0,000)$. Par contre, s'agissant des plantes herbacées, il n'existe pas une corrélation significative entre la variation du nombre mensuel d'espèces herbacées mellifères et celle de la richesse spécifique des plantes herbacées en fleurs $(r=0,560$ et $p=$ 0,058). Ces dernières augmentent considérablement en nombre au cours de la période de septembre à décembre alors qu'elles ne contribuent ni à la diminution ni à l'augmentation du nombre d'espèces de plantes butinées.

\section{DISCUSSION}

La connaissance des plantes mellifères au Bénin reste encore insuffisante et partielle. Le premier inventaire réel de plantes mellifères au Bénin est celui de Fohounfo (2002) qui a identifié 120 espèces butinées par les abeilles pendant la petite saison pluvieuse et la grande saison sèche dans le village Ewè à Kétou, en zone guinéenne. Depuis lors, des efforts de recensement des plantes mellifères se sont poursuivis avec Yédomonhan (2004) qui a identifié 92 espèces mellifères dans la forêt classée de la Lama et Dèmènou (2006) qui a dénombré 56 espèces ligneuses butinées par les abeilles à Manigri. La contribution exclusive du présent travail à la connaissance des plantes mellifères au Bénin est de 21 espèces. La richesse spécifique de la flore mellifère connue au Bénin passe ainsi de 188 espèces (Fohounfo, 2002; Yédomonhan, 2004; Dèmènou, 2006) à 209 espèces, soit 7,5\% de la flore du Bénin (Akoègninou et al., 2006).

La richesse spécifique des plantes mellifères représente $32,7 \%$ des plantes en fleurs au cours de la période d'observation. Ces résultats montrent que les abeilles opèrent une véritable sélection des espèces. $\mathrm{La}$ sélection est plus forte que celle constatée par Fohounfo (2002) et Yédomonhan (2004) en zone guinéenne au Bénin. Cette sélection est influencée par la morphologie florale, la phénologie et la composition floristique (Lobreau-Callen et Damblon, 1994). Le nombre d'espèces mellifères recensées est supérieur à celui obtenu (64 espèces) dans la même zone de transition soudano-guinéenne au Togo (Aloma, 2000). Par contre, il est plus faible que les valeurs trouvées en zone soudanienne au Burkina Faso et qui sont de 96 et 97 espèces respectivement en zone soudanienne sud et soudanienne nord (Nombré, 2003). La différence numérique de la richesse spécifique entre ces sites serait liée à leur composition floristique et à l'éthologie 
de butinage de l'abeille domestique. En effet, le cortège floristique montre 37 espèces communes à celui de la zone soudanienne. Certaines espèces en fleurs recensées sont mellifères en zone soudanienne (Nombré, 2003) alors qu'elles ne le sont pas dans la présente étude. Il s'agit par exemple de Piliostigma thonninigii, Cochlospermum planchoni, Gardenia erubescens, Gardenia ternifolia, Pterocarpus erinaceus, Wissadula amplissa, etc. Ceci confirme l'idée de De Layens et Bonnier (1997) qui ont affirmé qu'une espèce peut être mellifère dans une zone et ne pas l'être dans une autre zone. Les taxons sus-cités sont, pour la plupart, butinés en zone soudanienne pour leur nectar (Nombré, 2003). Selon de nombreux auteurs (Crane, 1990; O’Toole et Raw, 1991; De Layens et Bonnier, 1997; Fluri et al., 2001a, 2001b), la quantité de nectar produite dépend entre autres du climat, du sol et de l'état sanitaire de la plante.

Sur le plan de la diversité des familles, la prédominance des Leguminosae, des Combretaceae et des Rubiaceae n'est pas une particularité de la végétation environnante du rucher de Manigri, mais une caractéristique générale des formations végétales naturelles des zones soudano-guinéenne et soudanienne (Sawadogo, 1993; Aloma, 2000; Nombré, 2003). Dans les zones à emprises agricoles, les familles dominantes en espèces mellifères sont souvent les Asteraceae et les Poaceae (Bakenga et al., 2000; Fohounfo, 2002 ; Nguemo et al., 2004). La forte diversité des Leguminosae-Mimosoideae et des Combretaceae qui sont des familles à haute valeur mellifères (Guinko et al., 1992b) constitue un atout floristique intéressant pour la production du miel au niveau du rucher de Manigri en particulier et dans la zone soudano-guinéenne en général.

La flore mellifère est caractérisée par une forte diversité des plantes nectarifères $(31 \%)$ et des plantes mixtes $(35,6 \%)$. L'analyse des travaux d'inventaire visuel de plantes mellifères laisse apparaître un gradient croissant de richesse spécifique en plantes nectarifères de la zone guinéenne à la zone soudanienne en passant par la zone de transition soudano-guinéenne. C'est ainsi que le taux de plantes productrices de nectar passe de $47 \%$ (Yédomonhan, 2004) à 56,7\% (Fohounfo, 2002) en zone guinéenne, respectivement à $6^{\circ} 58^{\prime}$ et $7^{\circ} 28^{\prime}$ de latitude nord au Bénin. Il est de $66,7 \%$ en zone soudano-guinéenne à $8^{\circ} 54^{\prime}$ (présente étude) puis de $85,4 \%$ en zone soudanienne au Burkina Faso à $11^{\circ} 01^{\prime}$ de latitude nord (Nombré, 2003). Ceci confirme les observations de De Layens et Bonnier (1997) et de Fluri (2001a, 2001b) selon lesquelles la production de nectar dépend entre autres du climat et de la latitude. La végétation du rucher de Manigri offre donc plus de possibilités en ressources florales nectarifères $(66,7 \%)$ aux abeilles. Elle est, par conséquent, plus apte à l'apiculture que la végétation de la zone guinéenne au Bénin où le taux de plantes nectarifères est compris entre 47 et $56,7 \%$ (respectivement Yédomonhan, 2004 et Fohounfo, 2002). Cette potentialité mellifère du site est renforcée par la présence des 16 espèces (soit 18,4\%) qui peuvent être considérées comme espèces à haute valeur mellifère (tableau 1) suivant le double critère de Guinko et al. (1987).

L'évolution temporelle de la diversité des plantes en fleurs traduit une disponibilité permanente des ressources florales tout au long de l'année. Cette disponibilité est caractérisée par la prédominance des arbres et arbustes en saison sèche et des espèces herbacées en saison des pluies et en début de saison sèche. L'évolution du nombre d'espèces herbacées en fleurs au cours des mois est similaire aux résultats de Ramirez (2002) pour qui les plantes herbacées commencent leur floraison dès le démarrage des pluies, et le plus grand nombre d'espèces fleurissent en mi-saison pluvieuse ou en fin de saison de pluies. Le gradient de floraison observé fait que la richesse spécifique mensuelle des plantes mellifères n'évolue pas proportionnellement avec celle des plantes en fleurs. En effet, les espèces herbacées qui expliquent l'augmentation de la richesse spécifique des plantes en fleurs sont très peu butinées. Leur taux de sélection est de 13,8\% contre $85,7 \%$ pour les arbres et arbustes.

\section{Conclusion}

La végétation de Manigri est caractérisée par une diversité permanente de ressources florales. Cette diversité varie qualitativement et quantitativement en fonction des saisons. Au total, 87 espèces mellifères ont été recensées, représentant 
$32,7 \%$ des 266 espèces ayant fleuri. Les arbres et arbustes sont les plus butinés par les abeilles. Les plantes productrices de nectar sont plus nombreuses en zone soudanoguinéenne comparativement à la zone guinéenne. Ceci constitue alors un atout important pour la pratique de l'apiculture moderne dans la zone.

\section{REMERCIEMENTS}

Les auteurs remercient sincèrement la Fondation Internationale pour la Science (FIS) pour avoir financé cette recherche à travers la bourse B/4014-1.

\section{RÉFÉRENCES}

Agwu COC, Akambi TO. 1985. A palynological study of honey from four vegetation zones of Nigeria. Pollen and Spore, 17(3-4): 335-348.

Akoègninou $\mathrm{A}$, van der Burg $\mathrm{WJ}$, van der Maesen LJG. 2006. Flore Analytique du Bénin. Backhuys Publishers: Wageningen.

Aloma S. 2000. Etude des facteurs de production de miel dans deux zones écologiques différentes au sud du Togo: Les sites d'Aképé et d'Agotimé-Nyitoé. Mémoire de DEA, Université du Bénin, p.36.

Anonyme 2003. Troisième recensement général de la population et de l'habitation. Direction des Etudes Démographiques, INSAE-UNICEFUNFPA - DDC.

Bakenga M, Bahati M, Balagizi K. 2000. Inventaire des plantes mellifères de Bakavu et ses environs (Sud-Kivu, Est de la République Démocratique du Congo). Tropicultura, 18(2): 89-93.

Briane G. 1991. Cartographie des ressources mellifères dans les Pyrénées Centrales. Bull. Techn. Apic., 76: 163-170.

Crane E. 1990. Bees and Beekeeping. Science, Pratice and World Resources. Heinemann N. (ed). Oxford, UK.

Dèmènou BB. 2006. Inventaire des plantes mellifères et caractérisation pollinique des miels des élevages apicoles de la zone soudano-guinéenne de Manigri. Mémoire DIT, Université d'Abomey-Calavi (Bénin), 68p.

Dubroeucq D. 1977. Carte Pédologique de Reconnaissance de la République
Populaire du Bénin à 1/200 000 : Feuille Parakou. ORSTOM.

Faure P. 1977. Carte Pédologique de Reconnaissance de la République Populaire du Bénin à 1/200 000: Feuille Djougou. ORSTOM.

Fluri P, Pickhardt A, Cottier V, Charrière JD. 2001a. La pollinisation des plantes à fleurs par les abeilles. Biologie, Ecologie, Economie $1^{\text {ère }}$ partie. L'Abeille de France et l'Apiculteur, 871: 287-296.

Fluri P, Pickhardt A, Cottier V, Charrière JD. 2001b. La pollinisation des plantes à fleurs par les abeilles. Biologie, Ecologie, Economie $2^{\text {ème }}$ partie. L'Abeille de France et l'Apiculteur, 872: 335-340.

Fohounfo HT. 2002. Plantes mellifères et composition pollinique des miels de la petite saison des pluies et de la grande saison sèche au sud Bénin. Mémoire DIT, Université d'Abomey-Calavi (Bénin), 56.

Guinko S, Guenda W, Millogo-Rasolodimby J, Tamini Z, Zoungrana I. 1987. Etude des plantes mellifères dans l'Ouest du Burkina Faso (Province du Houet, de la Comoé et du Kénédougou). Rapport projet FAO TCP / BKF / 4510 (T).

Guinko S, Guenda W, Tamini Z, Zoungrana I. 1992a. Les plantes mellifères de la région ouest du Burkina Faso. Etudes flor. Veg. Burkina Faso, 1: 47-56.

Guinko S, Sawadogo M, Guenda W. 1992b. Etudes des plantes mellifères de saison pluvieuse et quelques aspects du comportement des abeilles dans la région de Ouagadougou, Burkina Faso. Etudes flor. Veg. Burkina Faso, 1: 27-46.

Houinato M. 2001. Phytosociologie, écologie, production et capacité de charge des formations végétales pâturées dans la région des Monts Kouffé (Bénin). Thèse de Doctorat. Université Libre de Bruxelles, $219 \mathrm{p}$.

Kepaletswe K. 1997. Beekeeping in Botswana (4th edn); $76 \mathrm{p}$.

De Layens G, Bonnier G. 1997. Cours complet d'Apiculture et Conduite d'un Rucher Isolé. Editions Belin.

Lobreau-Callen D, Darchen R, Le Thomas A. 1986. Apport de la palynologie à la connaissance des relations abeilles / plantes en savanes arborées du Togo et du Bénin. Apidologie, 17(4): 279-306. 
Lobreau-Callen D, Damblon F. 1994. Spectre pollinique des miels de l'abeille Apis mellifera L. (Hymenoptera, Apidae) et zones de végétation en Afrique occidentale tropicale et méditerranéenne. Grana, 33: 245-253.

Millogo-Rasolodimby J. 1989. Importance apicole du karité (Butyrospermum paradoxum (Gaern.) Hepper) et du néré (Parkia biglobosa (Jacq.) Benth.). Revue française d'Apiculture, 482: 72-75.

Nguemo DD, Foko J, Pinta JY, Ngouo LV, Tchoumboue J, Zango P. 2004. Inventaire et identification des plantes mellifères de la zone soudano-guinéenne d'altitude de l'ouest Cameroun. Tropicultura, 22(3): 139-145.

Nombré I. 2003. Etudes des potentialités mellifères de deux zones du Burkina Faso : Garango (province du Bouglou) et Nazinga (province du Nahouri). Thèse de Doctorat d'Université, Université de Ouagadougou, 156p.

O'Toole C, Raw A. 1991. Bees and flowers. In Bees of the World; 128-142.

Ramirez N. 2002. Reproductive phenology, life-forms, and habitats of the Venezuela
Central Plain. American Journal of Botany, 89(2): 836-842.

Sawadogo M. 1993. Contribution à l'étude du cycle des miellées et du cycle biologique annuel des colonies d'abeilles Apis mellifica adansonii Latr. à l'ouest du Burkina Faso. Thèse de Doctorat, Université de Ouagadougou, 152p.

Seeley TD 1985. Honeybee ecology, a study of adaptation in social life. Princeton University Press: Princeton, New Jersey, USA.

Tossou GM, Akoègninou A, Yédomonhan $\mathrm{H}$, Batawila K, Akpagana K. 2005. Analyse pollinique des miels de la forêt classée de la Lama (Bénin) et son apport à la connaissance de la flore apicole. J. Rech. Sci. Univ. Lomé (Togo), série A, 7(1): 8392.

Villières B. 1987. L'Apiculture en Afrique Tropicale, Dossier «Le point sur», 11, GERT, ACCT, AFVP : Paris.

Yédomonhan H. 2004. Plantes mellifères et miels du Bénin : cas de la forêt classée de la Lama. Mémoire de DEA, Université de Lomé (Togo), 65p. 